\title{
The major genetic risk factor for severe COVID-19 is inherited from Neanderthals
}

https://doi.org/10.1038/s41586-020-2818-3

Received: 3 July 2020

Accepted: 22 September 2020

Published online: 30 September 2020

Check for updates

\author{
Hugo Zeberg ${ }^{1,2 \bowtie} \&$ Svante Pääbo ${ }^{1,3 凶}$
}

A recent genetic association study ${ }^{1}$ identified a gene cluster on chromosome 3 as a risk locus for respiratory failure after infection with severe acute respiratory syndrome coronavirus 2 (SARS-CoV-2). A separate study (COVID-19 Host Genetics Initiative) ${ }^{2}$ comprising 3,199 hospitalized patients with coronavirus disease 2019 (COVID-19) and control individuals showed that this cluster is the major genetic risk factor for severe symptoms after SARS-CoV-2 infection and hospitalization. Here we show that the risk is conferred by a genomic segment of around 50 kilobases in size that is inherited from Neanderthals and is carried by around $50 \%$ of people in south Asia and around $16 \%$ of people in Europe.
The COVID-19 pandemic has caused considerable morbidity and mortality, and has resulted in the death of over a million people to date ${ }^{3}$. The clinical manifestations of the disease caused by the virus, SARS-CoV-2, vary widely in severity, ranging from no or mild symptoms to rapid progression to respiratory failure ${ }^{4}$. Early in the pandemic, it became clear that advanced age is a major risk factor, as well as being male and some co-morbidities 5 . These risk factors, however, do not fully explain why some people have no or mild symptoms whereas others have severe symptoms. Thus, genetic risk factors may have a role in disease progression. A previous study ${ }^{1}$ identified two genomic regions that are associated with severe COVID-19: one region on chromosome 3 , which contains six genes, and one region on chromosome 9 that determines ABO blood groups. Recently, a dataset was released by the COVID-19 Host Genetics Initiative in which the region on chromosome 3 is the only region that is significantly associated with severe COVID-19 at the genome-wide level (Fig. 1a). The risk variant in this region confers an odds ratio for requiring hospitalization of 1.6 (95\% confidence interval, 1.42-1.79) (Extended Data Fig. 1).

The genetic variants that are most associated with severe COVID19 on chromosome $3(45,859,651-45,909,024$ (hg19)) are all in high linkage disequilibrium (LD) - that is, they are all strongly associated with each other in the population $\left(r^{2}>0.98\right)$-and span 49.4 thousand bases (kb) (Fig. 1b). This 'core' haplotype is furthermore in weaker linkage disequilibrium with longer haplotypes of up to $333.8 \mathrm{~kb}\left(r^{2}>0.32\right)$ (Extended Data Fig. 2). Some such long haplotypes have entered the human population by gene flow from Neanderthals or Denisovans, extinct hominins that contributed genetic variants to the ancestors of present-day humans around 40,000-60,000 years ago ${ }^{6,7}$. We therefore investigated whether the haplotype may have come from Neanderthals or Denisovans.

The index variants of the two studies ${ }^{1,2}$ are in high linkage disequilibrium $\left(r^{2}>0.98\right)$ in non-African populations (Extended Data Fig. 3 ). We found that the risk alleles of both of these variants are present in a homozygous form in the genome of the Vindija 33.19 Neanderthal, an approximately 50,000-year-old Neanderthal from Croatia in southern Europe $^{8}$. Of the 13 single nucleotides polymorphisms constituting the core haplotype, 11 occur in a homozygous form in the Vindija 33.19
Neanderthal (Fig. 1b). Three of these variants occur in the Altai ${ }^{9}$ and Chagyrskaya $8^{10}$ Neanderthals, both of whom come from the Altai Mountains in southern Siberia and are around 120,000 and about 60,000 years old, respectively (Extended Data Table 1), whereas none of the variants occurs in the Denisovan genome ${ }^{11}$. In the 333.8-kb haplotype, the alleles associated with risk of severe COVID-19 similarly match alleles in the genome of the Vindija 33.19 Neanderthal (Fig. 1b). Thus, the risk haplotype is similar to the corresponding genomic region in the Neanderthal from Croatia and less similar to the Neanderthals from Siberia.

We next investigated whether the core 49.4-kb haplotype might be inherited by both Neanderthals and present-day people from the common ancestors of the two groups that lived about 0.5 million years ago? The longer a present-day human haplotype shared with Neanderthals is, the less likely it is to originate from the common ancestor, because recombination in each generation will tend to break up haplotypes into smaller segments. Assuming a generational time of 29 years ${ }^{12}$, the local recombination rate $^{13}(0.53 \mathrm{cM}$ per $\mathrm{Mb})$, a split between Neanderthals and modern humans of 550,000 years ${ }^{9}$ and interbreeding between the two groups around 50,000 years ago, and using a published equation ${ }^{14}$, we exclude that the Neanderthal-like haplotype derives from the common ancestor $(P=0.0009)$. For the 333.8-kb-long Neanderthal-like haplotype, the probability of an origin from the common ancestral population is even lower $\left(P=1.6 \times 10^{-26}\right)$. The risk haplotype thus entered the modern human population from Neanderthals. This is in agreement with several previous studies, which have identified gene flow from Neanderthals in this chromosomal region ${ }^{15-21}$ (Extended Data Table 2). The close relationship of the risk haplotype to the Vindija 33.19 Neanderthal is compatible with this Neanderthal being closer to the majority of the Neanderthals who contributed DNA to present-day people than the other two Neanderthals ${ }^{10}$.

A Neanderthal haplotype that is found in the genomes of the present human population is expected to be more similar to a Neanderthal genome than to other haplotypes in the current human population. To investigate the relationships of the 49.4-kb haplotype to Neanderthal and other human haplotypes, we analysed all 5,008 haplotypes in the 1000 Genomes Project ${ }^{22}$ for this genomic region. We included

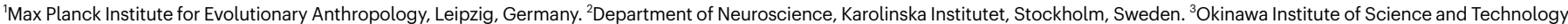

Onna-son, Japan. ${ }^{\bowtie}$-mail: hugo.zeberg@ki.se; paabo@eva.mpg.de 

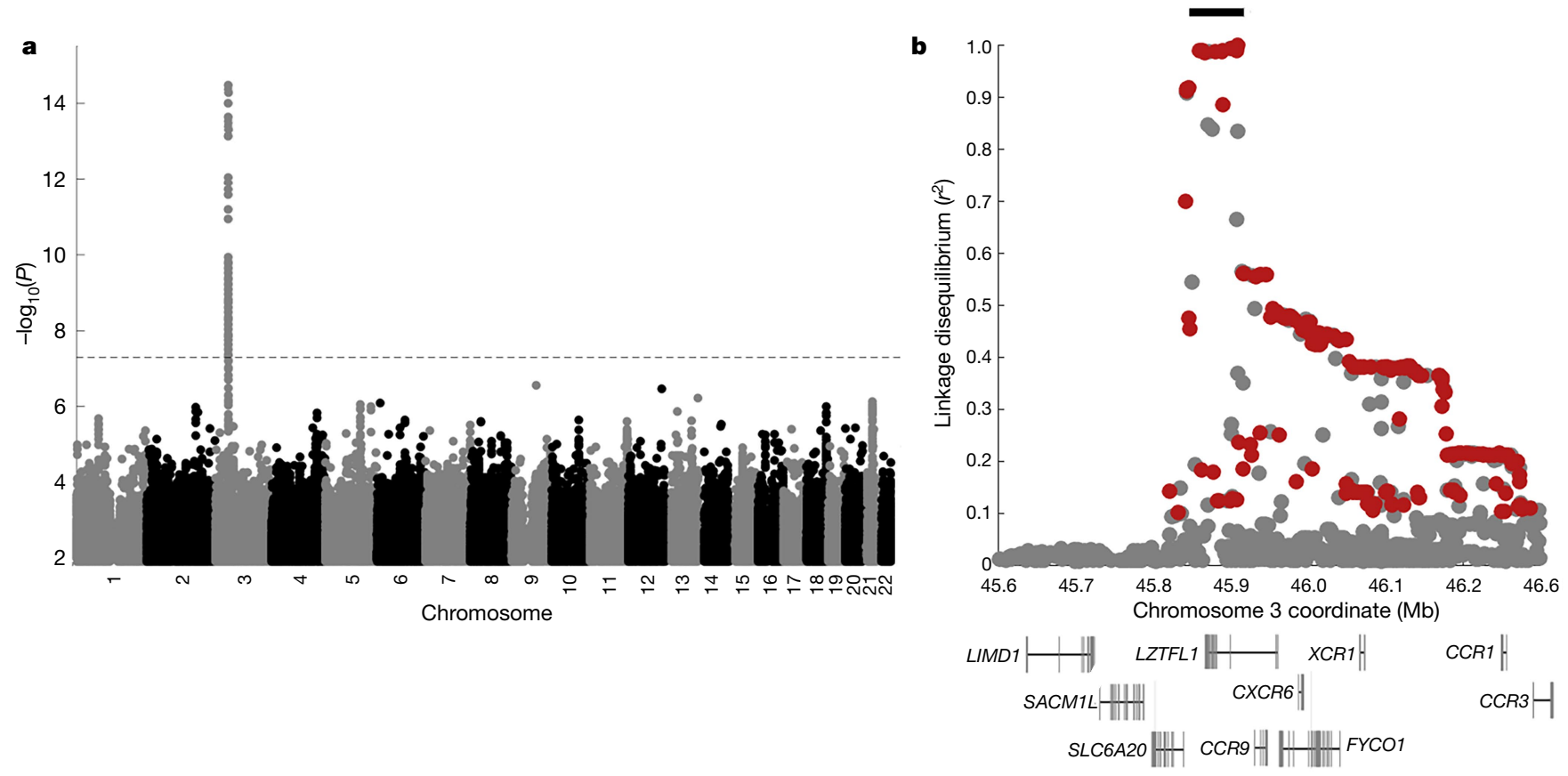

Fig. 1 Genetic variants associated with severe COVID-19. a, Manhattan plot of a genome-wide association study of 3,199 hospitalized patients with COVID-19 and 897,488 population controls. The dashed line indicates genomewide significance $\left(P=5 \times 10^{-8}\right)$. Data were modified from the COVID-19 Host

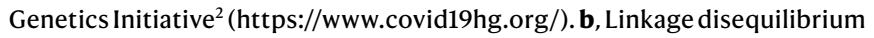
between the index risk variant (rs35044562) and genetic variants in the 1000

all positions that are called in the Neanderthal genomes and excluded variants found on only one chromosome and haplotypes seen only once in the 1000 Genomes Project data. This resulted in 253 present-day haplotypes that contained 450 variable positions. Figure 2 shows a phylogeny relating the haplotypes that were found more than 10 times (see Extended Data Fig. 4 for all haplotypes). We find that all risk haplotypes associated with severe COVID-19 form a clade with the three high-coverage Neanderthal genomes. Within this clade, they are most closely related to the Vindija 33.19 Neanderthal.

Among the individuals in the 1000 Genomes Project, the Neanderthal-derived haplotypes are almost completely absent from Africa, consistent with the idea that gene flow from Neanderthals into African populations was limited and probably indirect ${ }^{20}$. The Neanderthal core haplotype occurs in south Asia at an allele frequency of $30 \%$, in Europe at an allele frequency of $8 \%$, among admixed Americans with an allele frequency of $4 \%$ and at lower allele frequencies in east $\mathrm{Asia}^{23}$ (Fig. 3). In terms of carrier frequencies, we find that $50 \%$ of people in South Asia carry at least one copy of the risk haplotype, whereas $16 \%$ of people in Europe and $9 \%$ of admixed American individuals carry at least one copy of the risk haplotype. The highest carrier frequency occurs in Bangladesh, where more than half the population (63\%) carries at least one copy of the Neanderthal risk haplotype and $13 \%$ is homozygous for the haplotype. The Neanderthal haplotype may thus be a substantial contributor to COVID-19 risk in some populations in addition to other risk factors, including advanced age. In apparent agreement with this, individuals of Bangladeshi origin in the UK have an about two times higher risk of dying from COVID-19 than the general population ${ }^{24}$ (hazard ratio of $2.0,95 \%$ confidence interval, 1.7-2.4).

It is notable that the Neanderthal risk haplotype occurs at a frequency of $30 \%$ in south Asia whereas it is almost absent in east Asia (Fig. 3). This extent of difference in allele frequencies between south and east Asia is unusual $(P=0.006$, Extended Data Fig. 5$)$ and indicates that it may have been affected by selection in the past. Indeed, previous studies have
Genomes Project. Red circles indicate genetic variants for which the alleles are correlated to the risk variant $\left(r^{2}>0.1\right)$ and the risk alleles match the Vindija 33.19 Neanderthal genome. The core Neanderthal haplotype $\left(r^{2}>0.98\right)$ is indicated by a black bar. Some individuals carry longer Neanderthal-like haplotypes. The location of the genes in the region are indicated below using standard gene symbols. The $x$ axis shows hg19 coordinates.

suggested that the Neanderthal haplotype has been positively selected in Bangladesh ${ }^{25}$. At this point, we can only speculate about the reason for this-one possibility is protection against other pathogens. It is also possible that the haplotype has decreased in frequency in east Asia

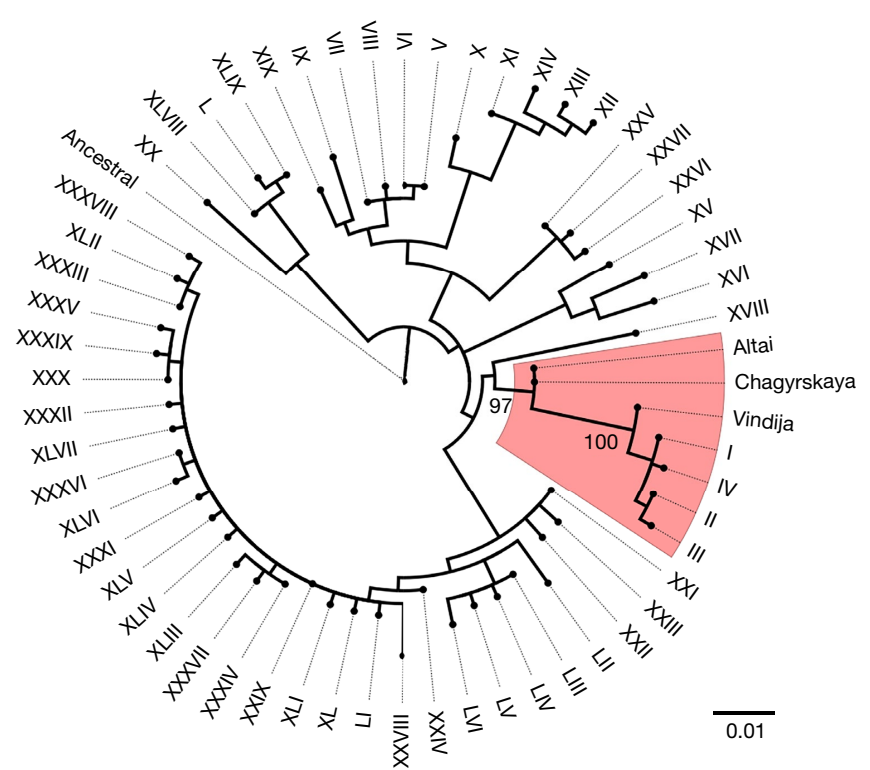

Fig. 2 | Phylogeny relating the DNA sequences that cover the core Neanderthal haplotype in individuals from the 1000 Genomes Project and Neanderthals. The coloured area highlights the haplotypes that carry the risk allele at rs35044562-that is, the risk haplotypes for severe COVID-19. Arabic numbers indicate bootstrap support (100 replicates). The phylogeny is rooted with the inferred ancestral sequence of present-day humans. The three Neanderthal genomes carry no heterozygous positions in this region. Scale bar, number of substitutions per nucleotide position. 


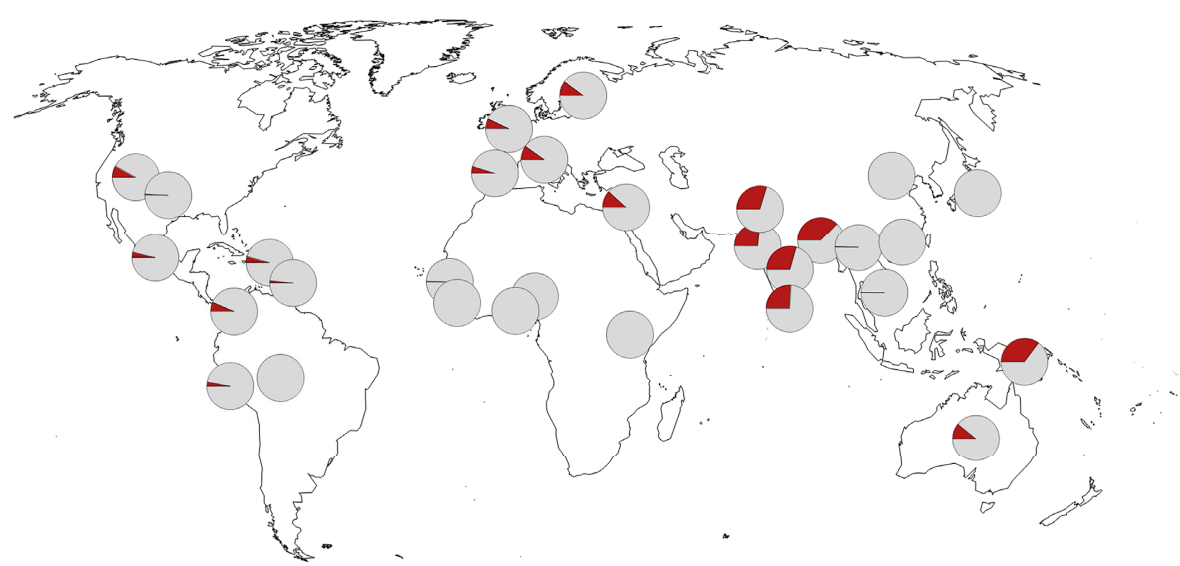

Fig. 3 | Geographical distribution of the Neanderthal core haplotype that confers risk for severe COVID-19. Pie charts show the minor allele frequency at rs35044562. Frequency data were obtained from the 1000 Genomes Project ${ }^{22}$. Map source data were obtained from OpenStreetMap ${ }^{23}$.

owing to negative selection, perhaps because of coronaviruses or other pathogens. In any case, the COVID-19 risk haplotype on chromosome 3 is similar to some other Neanderthal and Denisovan genetic variants that have reached high frequencies in some populations owing to positive selection or drift ${ }^{14,26-28}$, but it is now under negative selection owing to the COVID-19 pandemic.

It is currently not known what feature in the Neanderthal-derived region confers risk for severe COVID-19 and whether the effects of any such feature are specific to SARS-CoV-2, to other coronaviruses or to other pathogens. Once the functional feature is elucidated, it may be possible to speculate about the susceptibility of Neanderthals to relevant pathogens. However, with respect to the current pandemic, it is clear that gene flow from Neanderthals has tragic consequences.

\section{Online content}

Any methods, additional references, Nature Research reporting summaries, source data, extended data, supplementary information, acknowledgements, peer review information; details of author contributions and competing interests; and statements of data and code availability are available at https://doi.org/10.1038/s41586-020-2818-3.

1. Ellinghaus, D. et al. Genomewide association study of severe COVID-19 with respiratory failure. N. Engl. J. Med. https://doi.org/10.1056/NEJMoa2020283 (2020)

2. COVID-19 Host Genetics Initiative. The COVID-19 Host Genetics Initiative, a global initiative to elucidate the role of host genetic factors in susceptibility and severity of the SARS-CoV-2 virus pandemic. Eur. J. Hum. Genet. 28, 715-718 (2020).

3. WHO. Coronavirus disease (COVID-19) Weekly Epidemiological Update and Weekly Operational Update: Weekly Epidemiological Update 14 September 2020 https:// www.who.int/emergencies/diseases/novel-coronavirus-2019/situation-reports (2020).

Vetter, P. et al. Clinical features of COVID-19. Br. Med. J. 369, m1470 (2020).

5. Zhou, F. et al. Clinical course and risk factors for mortality of adult inpatients with COVID-19 in Wuhan, China: a retrospective cohort study. Lancet 395, 1054-1062 (2020).

6. Green, R. E. et al. A draft sequence of the Neandertal genome. Science 328, 710-722 (2010)

7. Sankararaman, S., Patterson, N., Li, H., Pääbo, S. \& Reich, D. The date of interbreeding between Neandertals and modern humans. PLoS Genet. 8, e1002947 (2012).

8. Prüfer, K. et al. A high-coverage Neandertal genome from Vindija Cave in Croatia. Science 358, 655-658 (2017)
9. Prüfer, K. et al. The complete genome sequence of a Neanderthal from the Altai Mountains. Nature 505, 43-49 (2014).

10. Mafessoni, F. et al. A high-coverage Neandertal genome from Chagyrskaya Cave. Proc. Natl Acad. Sci. USA 117, 15132-15136 (2020).

11. Meyer, M. et al. A high-coverage genome sequence from an archaic Denisovan individual. Science 338, 222-226 (2012).

12. Langergraber, K. E. et al. Generation times in wild chimpanzees and gorillas suggest earlier divergence times in great ape and human evolution. Proc. Natl Acad. Sci. USA 109, 15716-15721 (2012).

13. Kong, A. et al. A high-resolution recombination map of the human genome. Nat. Genet. 31, 241-247 (2002)

14. Huerta-Sánchez, E. et al. Altitude adaptation in Tibetans caused by introgression of Denisovan-like DNA. Nature 512, 194-197 (2014).

15. Sankararaman, S. et al. The genomic landscape of Neanderthal ancestry in present-day humans. Nature 507, 354-357 (2014).

16. Vernot, B. \& Akey, J. M. Resurrecting surviving Neandertal lineages from modern human genomes. Science 343, 1017-1021 (2014).

17. Vernot, B. et al. Excavating Neandertal and Denisovan DNA from the genomes of Melanesian individuals. Science 352, 235-239 (2016).

18. Steinrücken, M., Spence, J. P., Kamm, J. A., Wieczorek, E. \& Song, Y. S. Model-based detection and analysis of introgressed Neanderthal ancestry in modern humans. Mol. Ecol. 27, 3873-3888 (2018)

19. Gittelman, R. M. et al. Archaic hominin admixture facilitated adaptation to out-of-Africa environments. Curr. Biol. 26, 3375-3382 (2016).

20. Chen, L., Wolf, A. B., Fu, W., Li, L. \& Akey, J. M. Identifying and interpreting apparent Neanderthal ancestry in African individuals. Cell 180, 677-687 (2020).

21. Skov, L. et al. The nature of Neanderthal introgression revealed by 27,566 Icelandic genomes. Nature 582, 78-83 (2020).

22. The 1000 Genomes Project Consortium. A global reference for human genetic variation. Nature 526, 68-74 (2015)

23. OpenStreetMap. Planet OSM. https://planet.osm.org/ (2017)

24. Public Health England. COVID-19: Review of Disparities in Risks and Outcomes. https:// www.gov.uk/government/publications/covid-19-review-of-dis parities-in-risks-and-outcomes (2020).

25. Browning, S. R., Browning, B. L., Zhou, Y., Tucci, S. \& Akey, J. M. Analysis of human sequence data reveals two pulses of archaic Denisovan admixture. Cell 173, 53-61 (2018).

26. Dannemann, M., Andrés, A. M. \& Kelso, J. Introgression of Neandertal- and Denisovan-like haplotypes contributes to adaptive variation in human Toll-like receptors. Am. J. Hum. Genet. 98, 22-33 (2016).

27. Zeberg, H., Kelso, J. \& Pääbo, S. The Neandertal progesterone receptor. Mol. Biol. Evol. 37, 2655-2660 (2020)

28. Zeberg, H. et al. A Neanderthal sodium channel increases pain sensitivity in present-day humans. Curr. Biol. 30, 3465-3469 (2020).

Publisher's note Springer Nature remains neutral with regard to jurisdictional claims in published maps and institutional affiliations.

(c) The Author(s), under exclusive licence to Springer Nature Limited 2020 


\section{Methods}

Linkage disequilibrium was calculated using LDlink $4.1^{29}$ and alleles were compared to the archaic genomes ${ }^{8-11}$ using tabix ${ }^{30}$ (HTSlib 1.10). Haplotypes were constructed from the phase 3 release of the 1000 Genomes Project $^{22}$ as described. Phylogenies were estimated with phyML $3.3^{31}$ using the Hasegawa-Kishino-Yano- $85^{32}$ substitution model with a gamma shape parameter and the proportion of invariant sites estimated from the data. The probability of observing a haplotype of a particular length or longer owing to incomplete lineage sorting was calculated as previously described ${ }^{14}$. The inferred ancestral states at variable positions among present-day humans were taken from Ensembl ${ }^{33}$. The distribution of frequency differences of Neanderthal haplotypes between east and south Asia was computed by filtering diagnostic Neanderthal variants (fixed positions in the three high-coverage Neanderthal genomes and the Neanderthal allele missing in 108 Yoruba individuals) using a published introgression map $^{20}$, followed by pruning using PLINK1.90 ${ }^{34}$ ( $r^{2}$ cut-off of 0.5 in a sliding window of 100 variants) and allele frequency assessment in the 1000 Genomes Project. Maps displaying allele frequencies and linkage disequilibrium in different populations were made using Mathematica 11.0 (Wolfram Research) and OpenStreetMap data.

For the meta-analysis carried out by the COVID-19 Host Genetics Initiative ${ }^{2}$, participants consented and ethical approvals were obtained (https://www.covid19hg.org/partners/). The following eight studies contributed to the meta-analysis of hospitalization versus population controls: Genetic modifiers for COVID-19-related disease 'BelCovid' (Université Libre de Bruxelles, Belgium), Genetic determinants of COVID-19 complications in the Brazilian population 'BRACOVID' (University of Sao Paulo, Brazil), deCODE (deCODE Genetics, Iceland), FinnGen (Institute for Molecular Medicine Finland, Finland), GEN-COVID (University of Siena, Italy), Genes \& Health (Queen Mary University of London, UK), COVID-19-Host(age) (Kiel University and University Hospitals of Oslo and Schleswig-Holstein, Germany and Norway) and the UK Biobank (UK).

\section{Reporting summary}

Further information on research design is available in the Nature Research Reporting Summary linked to this paper.

\section{Data availability}

The summary statistics of the genome-wide association study that support the finding of this study are available from the COVID-19 Host Genetics Initiative (round 3, ANA_B2_V2: hospitalized patients with COVID-19 compared with population controls; https://www.covid19hg. $\mathrm{org} /$ ). The genomes used are available from the 1000 Genomes Project (phase 3 release, https://www.internationalgenome.org/) and the Max Planck Institute for Evolutionary Anthropology (Chagyrskaya, Altai and Vindija 33.19, http://cdna.eva.mpg.de/neandertal/). The ancestral alleles are available at Ensembl (release 100, https://www.ensembl. org/). Map data are from OpenStreetMap and available from https:// www.openstreetmap.org.

29. Machiela, M. J. \& Chanock, S. J. LDlink: a web-based application for exploring population-specific haplotype structure and linking correlated alleles of possible functional variants. Bioinformatics 31, 3555-3557 (2015).

30. $\mathrm{Li}, \mathrm{H}$. Tabix: fast retrieval of sequence features from generic TAB-delimited files. Bioinformatics 27, 718-719 (2011).

31. Guindon, S. et al. New algorithms and methods to estimate maximum-likelihood phylogenies: assessing the performance of PhyML 3.0. Syst. Biol. 59, 307-321 (2010).

32. Hasegawa, M., Kishino, H. \& Yano, T. Dating of the human-ape splitting by a molecular clock of mitochondrial DNA. J. Mol. Evol. 22, 160-174 (1985).

33. Yates, A. D. et al. Ensembl 2020. Nucleic Acids Res. 48, D682-D688 (2020).

34. Chang, C. C. et al. Second-generation PLINK: rising to the challenge of larger and richer datasets. Gigascience 4, 7 (2015).

Acknowledgements We thank the COVID-19 Host Genetics Initiative for making the data from the genome-wide association study available, and the Max Planck Society and the NOMIS Foundation for funding.

Author contributions H.Z. performed the haplotype analysis. H.Z. and S.P. jointly wrote the manuscript.

Competing interests The authors declare no competing interests.

Additional information

Supplementary information is available for this paper at https://doi.org/10.1038/s41586-020 2818-3.

Correspondence and requests for materials should be addressed to H.Z. or S.P.

Peer review information Nature thanks Tobias Lenz, Yang Luo and the other, anonymous, reviewer(s) for their contribution to the peer review of this work. Peer reviewer reports are available.

Reprints and permissions information is available at http://www.nature.com/reprints. 
Cases Controls

UK Biobank EUR

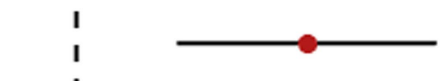

784

419,747

deCODE

BelCovid

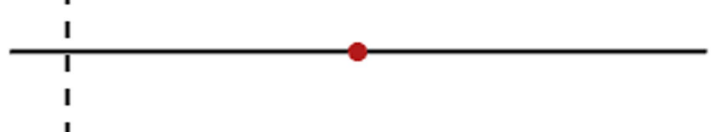

86

223,971

GENCOVID

HOST(age)

FinnGen

Genes \& Health

BRACOVID

COVID-19 HGI

(summary)

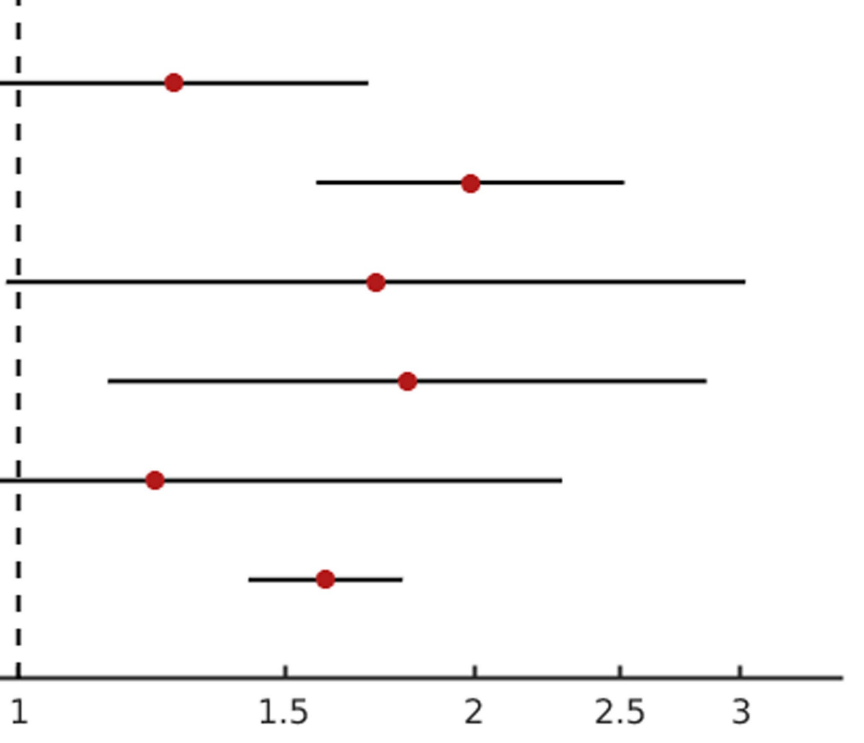

57

1,484

366

2,461

$1,610 \quad 2,205$

58

218,734

62

27,353

176

1,533

$3,199 \quad 897,488$

Odds ratio

Extended Data Fig. 1 | Odds ratios for hospitalization owing to COVID-19 for cohorts contributing to the meta-analysis (round 3) of the COVID-19 Host Genetics Initiative (rs35044562). The odds ratio and the $P$ value for the summary effect are odds ratio $=1.60$ (95\% confidence interval, $1.42-1.79)$ and $P=3.1 \times 10^{-15}$ (two-sided $z$-test, $n=3,199$ patients with COVID-19 and 897,488 controls over 8 independent studies). Data are the odds ratios and $95 \%$ confidence intervals. HOST(age), UK Biobank European (EUR), GENCOVID, deCODE and BelCovid use European population controls. BRACOVID, Genes \& Health and FinnGen use American, south Asian and Finnish population controls, respectively. 


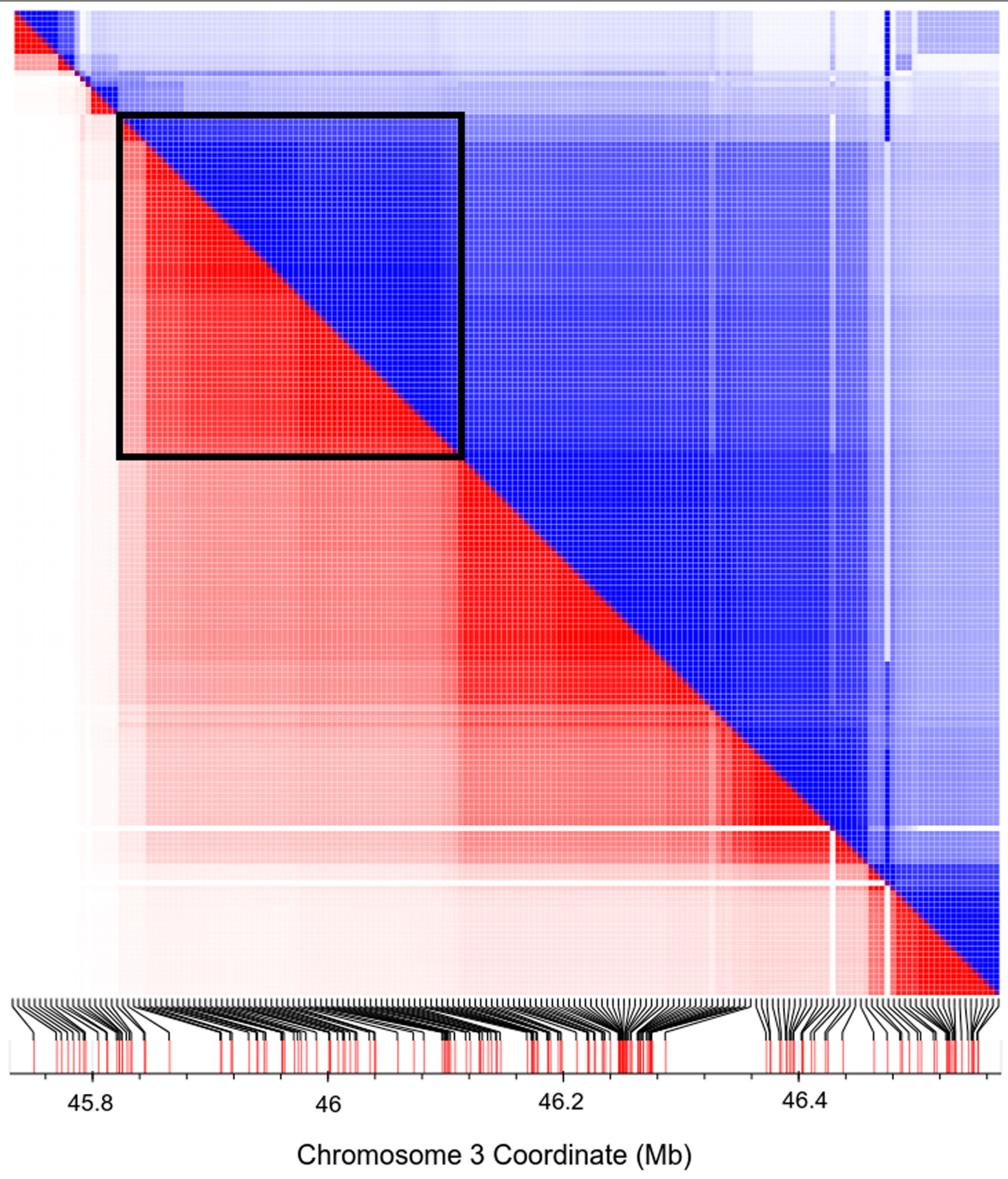

Correlation $\left(\mathbf{R}^{2}\right)$

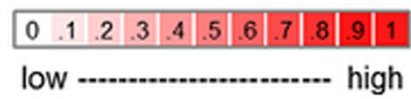

Extended Data Fig. 2 | Pairwise linkage disequilibrium between diagnostic Neanderthal variants. Heat map of linkage disequilibrium between genetic variants in which one allele is shared with three Neanderthal genomes and
Correlation (D')

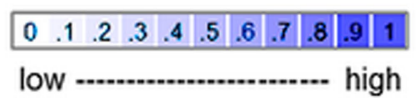

missing in 108 Yoruba individuals. The black box highlights a haplotype of 333.8 kb between rs17763537 and rs13068572 (chromosome 3: 45,843,315$46,177,096)$. Red, $r^{2}$ correlation; blue, $\mathrm{D}^{\prime}$ correlation. 
Article

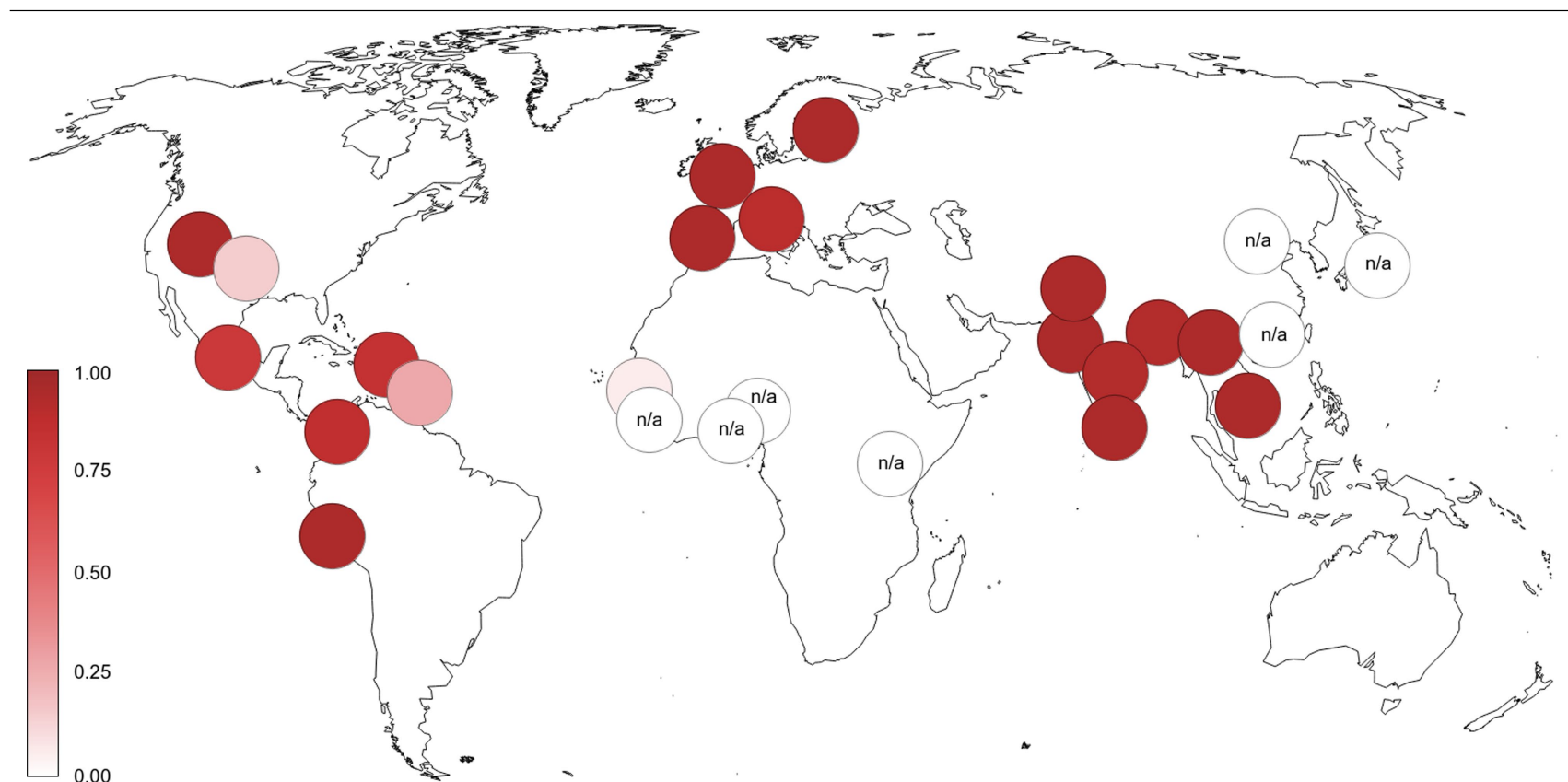

Extended Data Fig. 3 | Linkage disequilibrium between index variant rs11385942 and the index variant of the COVID-19 Host Genetics Initiative (rs35044562). Shades of red indicate the extent of linkage disequilibrium $\left(r^{2}\right)$ in the populations included in the 1000 Genomes Project. Populations labelled
' $\mathrm{n} / \mathrm{a}$ ' are monomorphic for the protective allele of rs 35044562. The previously described index variant $(\mathrm{rs} 11385942)^{1}$ does not have any genetic variants in linkage disequilibrium $\left(r^{2}>0.8\right)$ in populations from Africa. Map source data from OpenStreetMap ${ }^{23}$. 


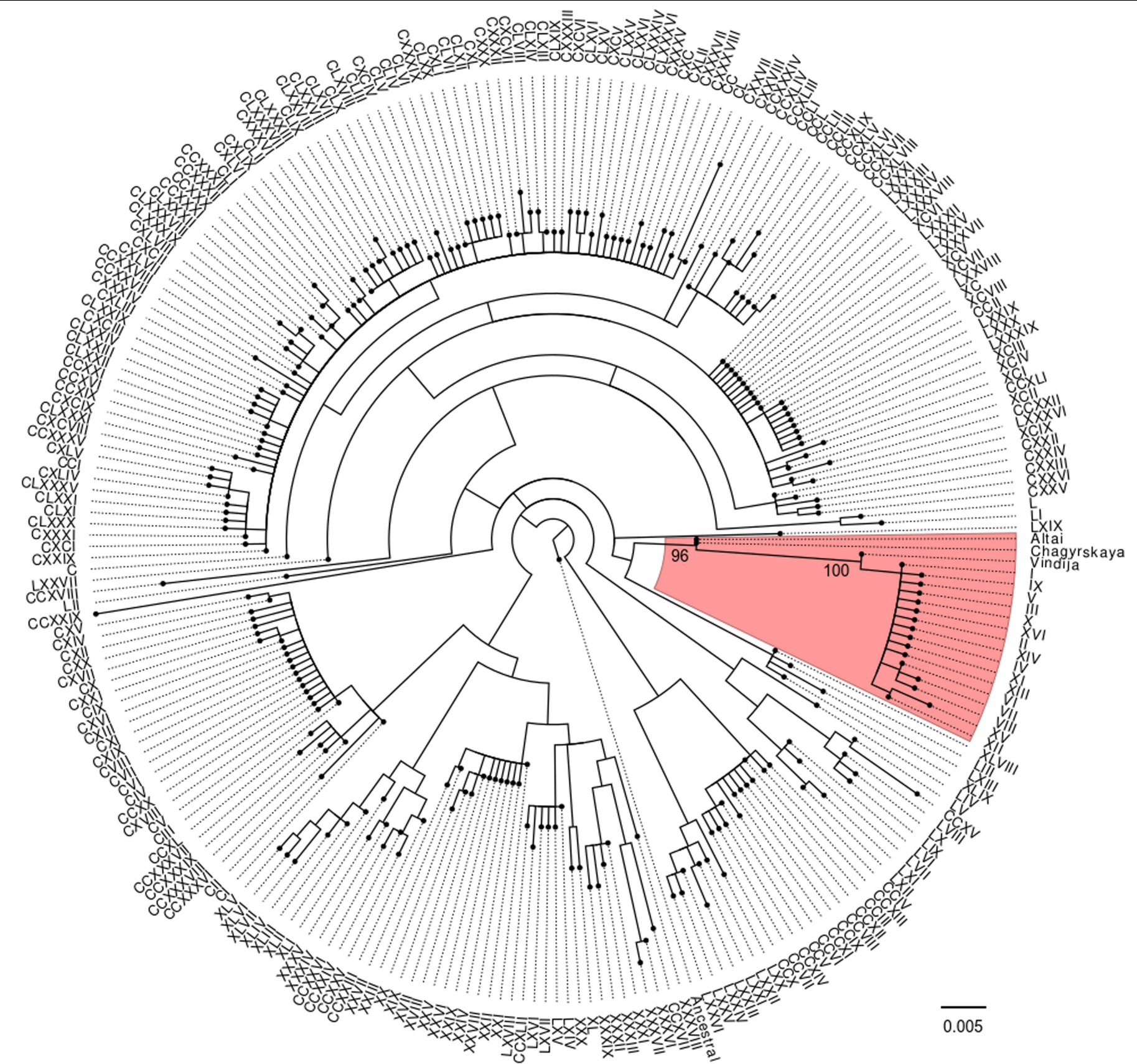

Extended Data Fig. 4 | Phylogeny of haplotypes in individuals included in the 1000 Genomes Project and Neanderthals covering the genomic region of the core risk haplotype. The shaded area highlights a monophyletic group that contains all present-day haplotypes carrying the risk allele at rs 35044562 and the haplotypes of the three high-coverage Neanderthals. Arabic numbers show bootstrap support (100 replicates). The tree is rooted with the inferred ancestral human sequence. Scale bar, number of substitutions per nucleotide position. 


\section{Article}

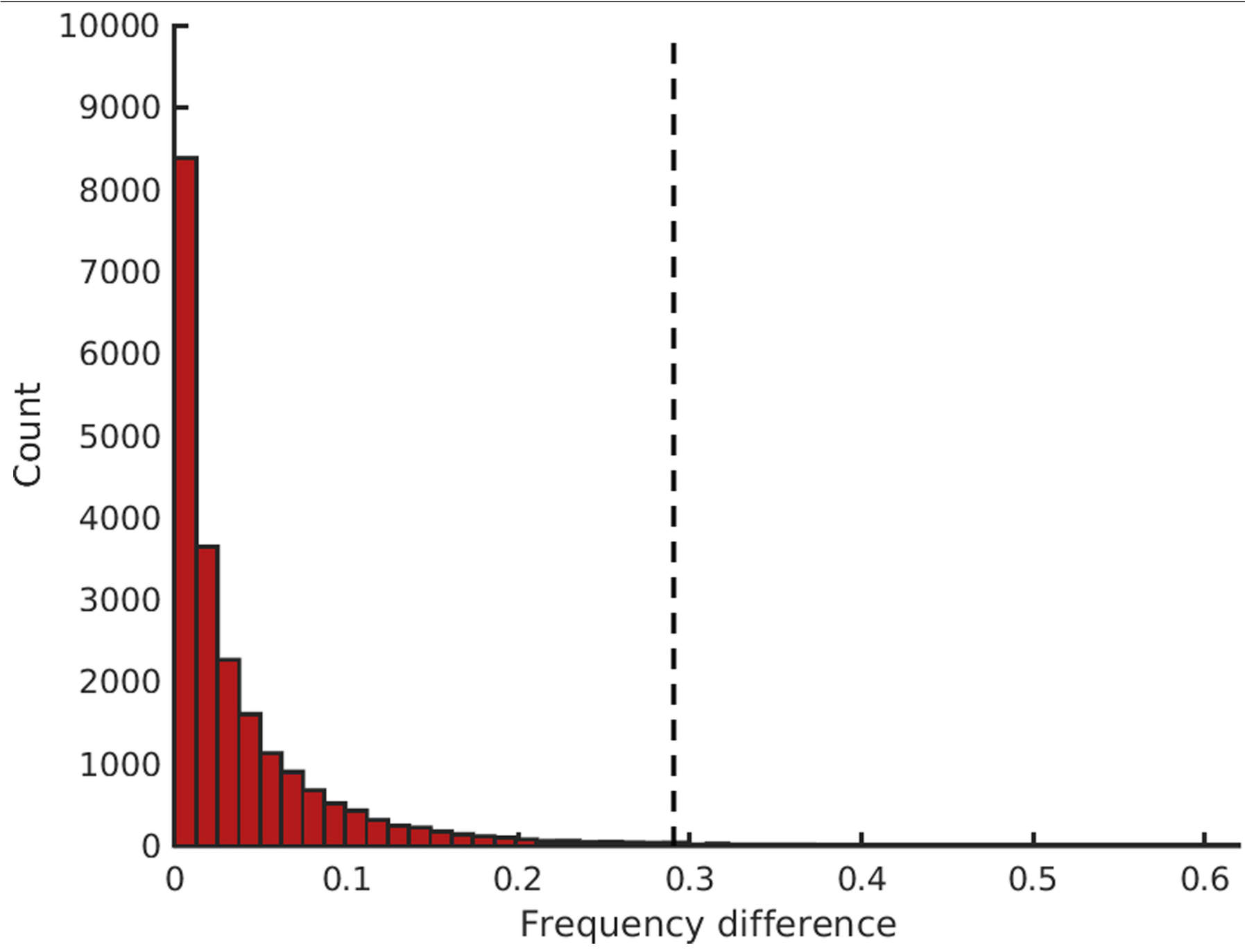

Extended Data Fig. 5 | Frequency differences between south and east Asia for haplotypes introgressed from Neanderthals. The dashed line indicates the frequency difference for the Neanderthal haplotype that confers risk of severe COVID-19. 
Extended Data Table 1 | Genetic variants in LD $\left(r^{2}>0.98\right)$ with $r s 35044562$ and the corresponding Neanderthal variants

\begin{tabular}{|c|c|c|c|c|c|c|c|c|}
\hline Chr & Pos & rsid & LD with rs35044562 & Ref & Alt/Risk & Vindija & Altai & Chagyrskaya \\
\hline 3 & 45909024 & rs35044562 & 1.000 & $A$ & $G$ & $\mathrm{G}$ & $\mathrm{G}$ & $\mathrm{G}$ \\
\hline 3 & 45901089 & rs73064425 & 0.992 & $\mathrm{C}$ & $\mathrm{T}$ & $\mathrm{T}$ & C & $\mathrm{C}$ \\
\hline 3 & 45899651 & rs34326463 & 0.992 & $A$ & $\mathrm{G}$ & $A$ & A & A \\
\hline 3 & 45908116 & rs13081482 & 0.989 & $A$ & $\mathrm{~T}$ & $\mathrm{~T}$ & $\mathrm{~T}$ & $\mathrm{~T}$ \\
\hline 3 & 45880481 & rs35508621 & 0.989 & $\mathrm{~T}$ & $\mathrm{C}$ & $\mathrm{C}$ & $\mathrm{T}$ & $\mathrm{T}$ \\
\hline 3 & 45864732 & rs10490770 & 0.989 & $\mathrm{~T}$ & $\mathrm{C}$ & $\mathrm{C}$ & $\mathrm{C}$ & $\mathrm{C}$ \\
\hline 3 & 45862952 & rs71325088 & 0.989 & $\mathrm{~T}$ & $\mathrm{C}$ & $\mathrm{C}$ & $\mathrm{T}$ & $\mathrm{T}$ \\
\hline 3 & 45861932 & rs13078854 & 0.989 & G & $A$ & $A$ & $G$ & G \\
\hline 3 & 45859651 & rs17713054 & 0.987 & G & A & A & $\mathrm{G}$ & $\mathrm{G}$ \\
\hline 3 & 45871908 & rs67959919 & 0.987 & G & $A$ & G & G & G \\
\hline 3 & 45888690 & rs34288077 & 0.987 & $A$ & $\mathrm{G}$ & $\mathrm{G}$ & $A$ & $A$ \\
\hline 3 & 45889921 & rs35081325 & 0.987 & $A$ & $\mathrm{~T}$ & $\mathrm{~T}$ & $A$ & $A$ \\
\hline 3 & 45867440 & rs35624553 & 0.984 & $A$ & G & G & $A$ & A \\
\hline
\end{tabular}

Data from the 1000 Genomes Project ${ }^{22}$. 'Ref' indicates the alleles from hg19. The three Neanderthal genomes are homozygous at these positions. LD, linkage disequilibrium. 


\section{Article}

Extended Data Table 2 | Previous studies that identified gene flow from Neanderthals at the core haplotype

\section{Study}

This study

Sankararaman et al. 2014

Vernot et al. 2014

Vernot et al. 2016

Gittelman et al. 2016

Steinrücken et al. 2018

Skov et al. 2020

Chen et al. 2020

Chr Start (Mb) Stop (Mb)

3

3

3

3

3

3

3

3
45.86

45.84

45.84

45.21

45.84

45.84

45.85

45.82
45.91

46.89

45.91

46.33

46.17

45.88

46.58

45.92 


\section{Reporting Summary}

Nature Research wishes to improve the reproducibility of the work that we publish. This form provides structure for consistency and transparency in reporting. For further information on Nature Research policies, see our Editorial Policies and the Editorial Policy Checklist.

\section{Statistics}

For all statistical analyses, confirm that the following items are present in the figure legend, table legend, main text, or Methods section.

n/a Confirmed

$\square$ The exact sample size $(n)$ for each experimental group/condition, given as a discrete number and unit of measurement

$\square \quad \boldsymbol{x}$ A statement on whether measurements were taken from distinct samples or whether the same sample was measured repeatedly

$\square \quad \boldsymbol{x}$ The statistical test(s) used AND whether they are one- or two-sided

$\boldsymbol{x}$ Only common tests should be described solely by name; describe more complex techniques in the Methods section.

$\mathbf{x} \square$ A description of all covariates tested

$\boldsymbol{x} \square$ A description of any assumptions or corrections, such as tests of normality and adjustment for multiple comparisons

x A full description of the statistical parameters including central tendency (e.g. means) or other basic estimates (e.g. regression coefficient)

$x$ AND variation (e.g. standard deviation) or associated estimates of uncertainty (e.g. confidence intervals)

For null hypothesis testing, the test statistic (e.g. $F, t, r$ ) with confidence intervals, effect sizes, degrees of freedom and $P$ value noted

- Give $P$ values as exact values whenever suitable.

$\boldsymbol{x} \square$ For Bayesian analysis, information on the choice of priors and Markov chain Monte Carlo settings

$\mathbf{x} \square$ For hierarchical and complex designs, identification of the appropriate level for tests and full reporting of outcomes

$\boldsymbol{x}$ Estimates of effect sizes (e.g. Cohen's $d$, Pearson's $r$ ), indicating how they were calculated

Our web collection on statistics for biologists contains articles on many of the points above.

\section{Software and code}

Policy information about availability of computer code

Data collection No new data was produced in the present study.

Data analysis LDlink 4.1 for linkage disequilibrium (LD), PhyML 3.3 for the maximum-likelihood phylogenies, tabix (HTSlib 1.10) for calling variants in the genomes. PLINK 1.90 for LD pruning. Mathematica 11.0 for creating maps. All software are publicly available and except Mathematica free of charge.

For manuscripts utilizing custom algorithms or software that are central to the research but not yet described in published literature, software must be made available to editors and reviewers. We strongly encourage code deposition in a community repository (e.g. GitHub). See the Nature Research guidelines for submitting code \& software for further information.

\section{Data}

Policy information about availability of data

All manuscripts must include a data availability statement. This statement should provide the following information, where applicable:

- Accession codes, unique identifiers, or web links for publicly available datasets

- A list of figures that have associated raw data

- A description of any restrictions on data availability

GWAS results (round 3, ANA_B2_V2): https://www.covid19hg.org/results/ Neandertal genomes (Altai, Vindija 33.19, Chagyrskaya): http://cdna.eva.mpg.de/neandertal/ 1000 genomes project (phase 3 release): https://www.internationalgenome.org/ Ensembl (release 100): http://www.ensembl.org/ 


\section{Field-specific reporting}

Please select the one below that is the best fit for your research. If you are not sure, read the appropriate sections before making your selection.

\section{Life sciences study design}

All studies must disclose on these points even when the disclosure is negative.

Sample size We used all available high-coverage Neandertal genomes ( $n=3)$. The sample size of the GWAS (3,199 cases and 897,488 controls) was limited by the data provided from the cohorts. We used all genomes in the phase 3 release $(n=2504)$ of the 1000 genomes project.

Data exclusions Sites which are not shared between any two individuals were excluded, since those positions in the genome are not informative for the phylogenetic relationship. This exclusion criterium was not pre-established.

Replication The findings in our study are easily reproducible using publicly available genomes. The significance of the phylogenies was assessed using bootstrap: the identified haplotypes grouped with the Vindija Neandertal 100 times out of 100 bootstrap replicates. All eight cohorts contributing to the meta-analysis showed a positive correlation between the risk allele and hospitalization.

Randomization We used all relevant public data at hand, hence we did not performed any randomization of a subsample or equivalent. The genetic association study is not the product of this manuscript, we have only interpreted the results in an evolutionary perspective. For the phylogenetic trees, however, we use the built-in random number generator of PhyML 3.3 to calculate the phylogenies. As stated above, all bootstrap replicates resulted in the same monophyletic group.

Blinding We analysed publicly available meta statistics from a genetic association study of hospitalized COVID-19 patients. The nature of the underlying data (hospitalized COVID-19 patients) is such that blinding (of hospitalization and infection with SARS-CoV2) was not possible within ethical and practical constraints.

\section{Reporting for specific materials, systems and methods}

We require information from authors about some types of materials, experimental systems and methods used in many studies. Here, indicate whether each material, system or method listed is relevant to your study. If you are not sure if a list item applies to your research, read the appropriate section before selecting a response.

Materials \& experimental systems

\begin{tabular}{l|l} 
Methods \\
\hline n/a & Involved in the study \\
\hline $\boldsymbol{x}$ & $\square$ ChIP-seq \\
\hline $\boldsymbol{x}$ & $\square$ Flow cytometry \\
$\boldsymbol{x}$ & $\square$ MRI-based neuroimaging
\end{tabular}

\begin{tabular}{l|l}
\hline $\mathrm{n} / \mathrm{a}$ & Involved in the study \\
\hline $\boldsymbol{x}$ & $\square$ Antibodies \\
\hline $\boldsymbol{x}$ & $\square$ Eukaryotic cell lines \\
\hline $\boldsymbol{x}$ & $\square$ Palaeontology and archaeology \\
\hline $\boldsymbol{x}$ & $\square$ Animals and other organisms \\
\hline $\boldsymbol{x}$ & $\square$ Human research participants \\
\hline $\mathbf{x}$ & $\square$ Clinical data \\
$\mathbf{x}$ & $\square$ Dual use research of concern
\end{tabular}

